\title{
Lucas Sayılarıyla İlişkili İki Parçalı Graflar
}

\author{
Ahmet ÖTELEŞ ${ }^{1 *}$, Diyar Omar Mustafa ZANGANA ${ }^{2}$ \\ ${ }^{1}$ Dicle Üniversitesi, Ziya Gökalp Eğitim Fakültesi, Matematik Bölümü, 21280, Diyarbakir, TÜRKIYE \\ ${ }^{2}$ Siirt Üniversitesi, Fen Edebiyat Fakültesi, Matematik Bölümü, 56100, Siirt, TÜRKIYE
}

Geliş / Received: 01/04/2020, Kabul / Accepted: 15/09/2020

\begin{abstract}
Öz
Bu çalışmada iki parçalı komşuluk matrisi $n \times n$ mertebeli $(0,1)$-circulant matrisi olan iki parçalı bir graf ele aldık. Daha sonra bu grafın mükemmel eşlemelerinin (1-factor) sayılarının Lucas sayıları ile arasındaki ilişkiyi verdik. Son olarak da bu mükemmel eşlemelerin sayısını hesaplamak için bazı maple prosedürleri verdik.
\end{abstract}

Anahtar Kelimeler: Mükemmel eşleme, İki parçalı graf, Permanent, Fibonacci sayısı, Lucas sayısı

\section{Bipartite Graphs Associated with Lucas Numbers}

\section{Abstract}

In this paper, we consider the bipartite graph whose bipartite adjacency matrix is an $n \times \mathrm{n}(0,1)$-circulant matrix. Then we show that the numbers of perfect matchings of this graph are equal to the well-known Lucas numbers. Finally, we give some Maple procedures in order to calculate the numbers of perfect matchings of the bipartite graph.

Keywords: Perfect matching, bipartite graph, permanent, Fibonacci number, Lucas number

\section{Giriş}

Fibonacci ve Lucas sayıları pozitif tamsayıların geniş bir ailesine aittir. Bilim ve sanatın hemen her alanında birçok ilginç özelliğe ve uygulamaya sahiptirler. Yeni keşifler için çok değerli firsatlar sunmaya devam etmekle birlikte matematiğin güzelliğine, özellikle de sayılar teorisine oldukça katkıda bulunmaktadırlar (Koshy, 2001; Koshy, 2011). Bu say1 dizileri ile ilgili çok sayıdaki çalışmalardan bazılarına (Lee, 2000; Shiu ve Lam, 2003; Kılıç ve Taşç1, 2008; Özkan vd., 2018; Özkan ve Altun,
2019; Özkan ve Taştan, 2019) kayanaklarından ulaşılabilir.

Meşhur Fibonacci $\left\{F_{n}\right\}_{n=0}^{\infty}$ ve Lucas $\left\{L_{n}\right\}_{n=0}^{\infty}$ dizileri, $n \geq 2$ için

$$
\begin{aligned}
& F_{n}=F_{n-1}+F_{n-2}, \quad F_{0}=0 \text { ve } F_{1}=1 \\
& L_{n}=L_{n-1}+L_{n-2}, \quad L_{0}=2 \text { ve } L_{1}=1
\end{aligned}
$$

şeklindeki rekürans bağıntısıyla tanımlanırlar (Koshy, 2001).

Fibonacci ve Lucas dizileri "The OnLine Encyclopedia of Integer Sequences"

\footnotetext{
*Corresponding Author: aoteles@ dicle.edu.tr
} 
sayfasinda sirasiyla A000045 ve A000032 şeklinde adlandırılırlar (OEIS, 2013).

$$
\begin{aligned}
n \geq 1 \text { için } \\
L_{n}=F_{n-1}+F_{n+1}=F_{n}+2 F_{n-1}
\end{aligned}
$$

şeklinde tanımlanan rekürans bağıntısı, Fibonacci ve Lucas sayllarının birbirleriyle ilişkisini veren önemli bir bağıntıdır (Koshy, 2001).

Fibonacci ve Lucas sayılarının bazı değerlerini Tablo 1'de görebiliriz.

Tablo 1. Fibonacci ve Lucas Sayılarının Bazı Değerleri

\begin{tabular}{c|ccccccccccccc}
$n$ & 0 & 1 & 2 & 3 & 4 & 5 & 6 & 7 & 8 & 9 & 10 & 11 & $\cdots$ \\
\hline$F_{n}$ & 0 & 1 & 1 & 2 & 3 & 5 & 8 & 13 & 21 & 34 & 55 & 89 & $\cdots$ \\
$L_{n}$ & 2 & 1 & 3 & 4 & 7 & 11 & 18 & 29 & 47 & 76 & 123 & 199 & $\cdots$
\end{tabular}

İki parçalı grafların özelliklerinin araştırılması König tarafından başlatılmıştır. Matrislerin determinantları yardımıyla komşuluk matrislerinin özelliklerinin incelenmesi ile birlikte iki parçalı graflarla ilgili teorik açıdan yeni bir yaklaşım ortaya çıkmıştır (König, 1915; König, 1916). Uygulamalı bir konu olarak ise iki parçalı graflar, sosyal ağ analizi, demiryolu optimizasyonu problemi, evlilik problemi vb. gibi iki farklı nesne türü arasındaki etkileşimin bir modelini oluşturur (Asratian vd., 1998).

İki parçalı grafların mükemmel eşlemelerinin gerçek yapımı veya sayımı, yöneylem araştırmasında ortaya çıkan atama ve çizelgeleme problemleri ve maksimum akış problemleri gibi uygulamalara sahiptir (Minc, 1978). İki parçalı grafların mükemmel eşlemelerinin sayısının organik kimyada da ayrıca önemli bir rolü vardır (Wheland, 1953).

$G$ bir graf ve $V$ de $V_{1}$ ve $V_{2}$ gibi iki altkümeye ayrılmış $G$ 'nin düğümler kümesi olsun. Eğer $G$ 'nin her kenarı $V_{1}$ 'deki bir dügüümü $V_{2}$ 'deki bir düğüme bağlıyorsa $G^{\prime}$ ye iki parçalı graf denir. Bir grafta her düğüm tam olarak tek bir kenar ile bağlı ise bu eşlemeye mükemmel eşleme (1-factor) denir. Başka bir deyişle, her bir düğümün derecesi 1'dir. $A(G)$ iki parçalı bir $G$ grafının komşuluk matrisi ve $\mu(G)$ de iki parçalı $G$ grafının mükemmel eşlemelerinin bir sayısı olsun. Buna göre $\mu(G)$ ve $A(G)$ arasındaki ilişkiyi veren bağıntı $\mu(G)=\sqrt{\operatorname{per}(A(G))} \quad$ şeklindedir (Minc, 1978).

$G$ iki parçalı graf ve $V$ de $\left|V_{1}\right|=\left|V_{1}\right|=n \in$ $\mathbb{N}$ olmak üzere $V_{1}$ ve $V_{2}$ gibi iki altkümeye ayrılmış G'nin düğümler kümesi olsun. Ayrıca $G$ 'nin iki parçalı komşuluk matrisi $B(G)=\left(b_{i j}\right)$ de eğer $G$ grafı $v_{i} \in V_{1}$ 'den $v_{j} \in V_{2}$ 'ye bir kenar içeriyorsa $b_{i j}=1$, aksi taktirde $b_{i j}=0$ şeklinde tanımlansın. $\mathrm{Bu}$ durumda, $G$ iki parçalı grafin mükemmel eşlemelerinin sayısı, onun iki parçalı komşuluk matrisinin permanentine eşittir (Minc, 1978).

$S_{n} \quad\{1,2,3,4, \ldots, n\} \quad$ kümesinin bütün $n$ ! sayıdaki $\sigma$ permütasyonlarının bir simetrik grubu olsun. Buna göre $n \times n$ mertebesindeki bir $A=\left[a_{i j}\right]$ matrisinin permanenti

$$
\operatorname{per}(A)=\sum_{\sigma \in S_{n}} \prod_{i=1}^{n} a_{i, \sigma(i)}
$$

şeklinde tanımlanır. Bir matrisin permanenti o matrisin determinantina benzer. Aradaki 
tek fark determinantın Laplace açılımınındaki minörlerin işaretinin pozitif olmasıdır (Minc, 1978). Permanentlerle ilgili temel bilgilere ve daha fazla uygulamalara (Marcus ve Minc, 1965; Harary, 1969; Brualdi ve Cvetkovic, 2009) kaynaklarından ulaşılabilir.

Permanentlerin fizik, kimya, mühendislik gibi birçok alanda uygulamaların olmasının yanında en önemlilerinden bazıları da graf teorisiyle ilgilidir. Bir grafın mükemmel eşlemelerinin sayısını hesaplama problemi ise graf teorisindeki diğer uygulamalara göre daha zahmetlidir (Minc, 1978). Dolayısıyla iki parçalı grafların mükemmel eşlemelerinin sayısını hesaplamak, son yıllarda ilgi gören bir problem olmuştur.

Araştırmacılar, iki parçalı grafların mükemmel eşlemelerinin sayısı ile meşhur tamsayı dizileri arasındaki ilişkiyi veren çalışmalardan bazılarını (Lee ve Lee, 1995; Lee vd., 1997; Lee, 2000; Shiu ve Lam, 2003; Kılıç ve Taşçı, 2007; Kılıç ve Taşçı, 2008; Kılıç ve Stakhov, 2009; Yılmaz ve Bozkurt, 2012; Fonseca vd., 2015; Öteleş, 2017) kaynaklarından bulabilirler.

$\mathrm{Bu}$ çalışmada ilk olarak Fibonacci sayılarıyla ilişkili iki parçalı graflar için bazı lemmalar verdik. Daha sonra bu lemmalardan yararlanarak, iki parçalı komşuluk matrisi $(0,1)$-circulant matrisi olan iki parçalı grafin mükemmel eşlemelerinin sayısının Lucas sayılarıyla ilişki olduğunu gösterdik. Son olarak bu grafın mükemmel eşlemelerinin saysısını hesaplamak için bazı maple prosedürleri verdik.

\section{Bulgular}

$A=\left[a_{i j}\right]$ matrisi, satır vektörleri $r_{1}, r_{2}, \ldots r_{m}$ olan $m \times n$ mertebeli bir matris olsun. Eğer $A$ matrisinin $k$. sütünununda diğer elemanları sıfır olmak şartıyla tam olarak sıfırdan farklı iki eleman varsa o zaman $A$ matrisine $k$. sütun üzerinden contraction (büzüşme) yapılabilir. Kabul edelim ki, $i \neq j$ ve $a_{i k} \neq 0 \neq a_{j k}$ olmak üzere $A$ matrisi $k$. sütuna göre contraction yapılabilir olsun. Buna göre, $A$ matrisinden $j$. satır ve $k$. sütun silinip $i$. satır yerine de $a_{j k} r_{i}+a_{i k} r_{k}$ vektörü yazılarak elde edilen $(m-1) \times(n-1)$ mertebeli $A_{i j: k}$ matrisine $i$. ve $j$. satıra bağlı olarak $k$. sütuna göre $A$ matrisinin contraction'u denir. Ĕger $i \neq j$ ve $a_{k i} \neq 0 \neq$ $a_{k j}$ olmak üzere $k$. satıra göre contraction yapılabilir ise $A_{k: i j}=\left[A_{i j: k}^{T}\right]^{T}$ matrisine $i$. ve $j$. sütuna bağlı olarak $k$. satıra göre $A$ matrisinin contraction'u denir. $r=1, \ldots, t$ için $A_{r}$ matrisi $A_{r-1}$ matrisinin bir contraction'u olsun. Buna göre $A_{0}=A$ ve $A_{t}=B$ olacak şekilde $A_{0}, A_{1}, \ldots A_{t}(t \geq 1)$ matrisleri varsa veya $B=A$ ise $A$ matrisi $B$ matrisine contract edilebilir denir (Brualdi ve Gibson, 1977).

Lemma 1.1. $n>1$ olmak üzere $A n \times n$ mertebeli negatif olmayan bir integral matris olsun. Eğer $B$ matrisi $A$ matrisinin bir contraction'u ise o zaman

$$
\operatorname{per} A=\operatorname{per} B
$$

dir (Brualdi ve Gibson, 1977).

Lee vd. (1997) iki parçalı komşuluk matrisi elemanlar1

$$
\mathrm{a}_{i, j}= \begin{cases}1, & |j-i| \leq 1 \text { ise } \\ 0, & \text { aksi taktirde }\end{cases}
$$

şeklinde tanımlanan 


$$
A(n)=\left[\begin{array}{ccccc}
1 & 1 & 0 & \cdots & 0 \\
1 & 1 & 1 & \ddots & \vdots \\
0 & 1 & 1 & \ddots & 0 \\
\vdots & \ddots & \ddots & \ddots & 1 \\
0 & \cdots & 0 & 1 & 1
\end{array}\right]
$$

formundaki $n$-kare $(0,1)$-tridagonal matrisi olan $G(A(n))$ iki parçalı grafını ele aldılar ve bu grafla ilgili aşağıdaki sonucu verdiler.

Lemma 2.1 $G(A(n))$, (2) ile verilen $A(n)$ iki parçalı komşuluk matrisinin iki parçalı grafi olsun. Bu taktirde $G(A(n))$ iki parçalı grafın mükemmel eşlemelerinin sayısı $(n+1)$-inci Fibonacci sayısı $F_{n+1}$ 'e eşittir.

Şimdi Lemma 2.2 ve Lemma 2.3'ü verelim.

Lemma 2.2. $G(U(n))$, iki parçalı komşuluk matrisi

$$
U(n)=\left[\begin{array}{cccccc}
1 & 0 & \cdots & 0 & 0 & 1 \\
1 & 1 & 0 & 0 & \cdots & 0 \\
1 & 1 & 1 & 0 & \ddots & \vdots \\
0 & 1 & 1 & \ddots & \ddots & 0 \\
\vdots & \ddots & \ddots & \ddots & 1 & 0 \\
0 & \cdots & 0 & 1 & 1 & 1
\end{array}\right]
$$

şeklinde tanımlanan iki parçalı graf olsun. Buna göre $G(U(n))$ iki parçalı grafin mükemmel eşlemelerinin sayısı $F_{n}+1$ 'e eşittir. Burada $F_{n}, n$-inci Fibonacci sayısıdır.

İspat. $1 \leq k \leq n-3$ olmak üzere $U^{k}(n)$, $U(n)$ matrisinin $k$-inc1 contraction'u olsun. $U(n)$ 'nin tanımına göre bu matrisin son sütununa contraction metodu uygulanabilir. O zaman $U(n)$ 'nin 1-inci contraction'ununu

$$
U^{1}(n)=\left[\begin{array}{cccccc}
1 & 0 & \cdots & 0 & 1 & 1 \\
1 & 1 & 0 & 0 & \cdots & 0 \\
1 & 1 & 1 & 0 & \ddots & \vdots \\
0 & 1 & 1 & \ddots & \ddots & 0 \\
\vdots & \ddots & \ddots & \ddots & 1 & 0 \\
0 & \cdots & 0 & 1 & 1 & 1
\end{array}\right]
$$

şeklinde elde ederiz. Benzer şekilde $U^{1}(n)$ matrisinin de son sütununa contraction metodu uygularsak

$$
U^{2}(n)=\left[\begin{array}{cccccc}
1 & 0 & \cdots & 0 & 1 & 2 \\
1 & 1 & 0 & 0 & \cdots & 0 \\
1 & 1 & 1 & 0 & \ddots & \vdots \\
0 & 1 & 1 & \ddots & \ddots & 0 \\
\vdots & \ddots & \ddots & \ddots & 1 & 0 \\
0 & \cdots & 0 & 1 & 1 & 1
\end{array}\right]
$$

matrisini elde ederiz. $F_{2}=1$ ve $F_{3}=2$ olduğundan bu değerleri yukarıdaki $U^{2}(n)$ matrisinde yazarsak bu matrisi

$$
U^{2}(n)=\left[\begin{array}{cccccc}
1 & 0 & \cdots & 0 & F_{2} & F_{3} \\
1 & 1 & 0 & 0 & \cdots & 0 \\
1 & 1 & 1 & 0 & \ddots & \vdots \\
0 & 1 & 1 & \ddots & \ddots & 0 \\
\vdots & \ddots & \ddots & \ddots & 1 & 0 \\
0 & \cdots & 0 & 1 & 1 & 1
\end{array}\right]
$$

şeklinde yazabiliriz. Yukarıdaki matrisin son sütununa contraction metodu tekrar uygulanırsa

$$
U^{3}(n)=\left[\begin{array}{cccccc}
1 & 0 & \cdots & 0 & F_{3} & F_{4} \\
1 & 1 & 0 & 0 & \cdots & 0 \\
1 & 1 & 1 & 0 & \ddots & \vdots \\
0 & 1 & 1 & \ddots & \ddots & 0 \\
\vdots & \ddots & \ddots & \ddots & 1 & 0 \\
0 & \cdots & 0 & 1 & 1 & 1
\end{array}\right]
$$

matrisini elde ederiz. $\mathrm{Bu}$ işlemlere devam ederek, $U(n)$ matrisinin $k$-inc1 contraction'u $1 \leq k \leq n-3$ için

$$
U^{k}(n)=\left[\begin{array}{cccccc}
1 & 0 & \cdots & 0 & F_{k} & F_{k+1} \\
1 & 1 & 0 & 0 & \cdots & 0 \\
1 & 1 & 1 & 0 & \ddots & \vdots \\
0 & 1 & 1 & \ddots & \ddots & 0 \\
\vdots & \ddots & \ddots & \ddots & 1 & 0 \\
0 & \cdots & 0 & 1 & 1 & 1
\end{array}\right]
$$

şeklinde elde ederiz. $U^{k}(n)$ matrisinde $k=n-3 \quad$ yazarsak, $\quad U(n) \quad$ matrisinin $(n-3)$-üncü contraction'u 


$$
U^{n-3}(n)=\left[\begin{array}{ccc}
1 & F_{n-3} & F_{n-2} \\
1 & 1 & 0 \\
1 & 1 & 1
\end{array}\right]
$$

şeklinde elde ederiz. Son sütuna göre $U^{n-3}(n)$ matrisinin contraction'u

$$
\begin{aligned}
U^{n-2}(n) & =\left[\begin{array}{cc}
F_{n-2}+1 & F_{n-2}+F_{n-3} \\
1 & 1
\end{array}\right] \\
& =\left[\begin{array}{cc}
F_{n-2}+1 & F_{n-1} \\
1 & 1
\end{array}\right]
\end{aligned}
$$

şeklinde buluruz. Lemma 1' den

$$
\begin{aligned}
\operatorname{per} U(n) & =\operatorname{per} U^{n-2}(n) \\
& =F_{n-1}+F_{n-2}+1 \\
& =F_{n}+1
\end{aligned}
$$

olur ki bu da istenendir.

Lemma 2.3. $G(V(n))$, iki parçalı komşuluk matrisi

$$
V(n)=\left[\begin{array}{cccccc}
1 & 0 & 0 & \cdots & 0 & 1 \\
1 & 1 & 1 & 0 & \cdots & 0 \\
0 & 1 & 1 & 1 & \ddots & \vdots \\
\vdots & \ddots & 1 & \ddots & \ddots & 0 \\
\vdots & & \ddots & \ddots & 1 & 1 \\
0 & \cdots & \cdots & 0 & 1 & 1
\end{array}\right]
$$

şeklinde tanımlanan iki parçalı graf olsun. Buna göre $G(V(n))$ iki parçalı grafın mükemmel eşlemelerinin sayısı $F_{n}+1$ 'e eşittir. Burada $F_{n}, n$-inci Fibonacci sayısıdır.

İspat. $\mathrm{Bu}$ lemma, $V(n)$ matrisinin 1-inci sütununa göre contraction metodu uygulanarak ispatlanabilir.

Teorem 2.4. $G(W(n))$, iki parçalı komşuluk matrisi

$$
W(n)=\left[\begin{array}{cccccc}
1 & 1 & 0 & \cdots & 0 & 1 \\
1 & 1 & 1 & \ddots & & 0 \\
0 & 1 & 1 & 1 & \ddots & \vdots \\
\vdots & \ddots & 1 & \ddots & \ddots & 0 \\
0 & & \ddots & \ddots & 1 & 1 \\
1 & 0 & \cdots & 0 & 1 & 1
\end{array}\right]
$$

şeklinde circulant matrisi ile tanımlanan iki parçalı graf olsun. Buna göre $G(W(n))$ iki parçalı grafın mükemmel eşlemelerinin sayısı $L_{n}+2$ 'e eşittir. Burada $L_{n}, n$-inci Lucas sayısidır.

İspat. $A(n), U(n)$ ve $V(n)$ sirasiyla (2), (3) ve (4)'deki matrisler olmak üzere, $W(n)$ matrisinin 1-inci sütununa göre Laplace metodunu uygularsak

$$
\begin{aligned}
\operatorname{per} W(n)= & \operatorname{per} A(n-1)+\operatorname{per} U(n-1) \\
& +\operatorname{per} V(n-1)
\end{aligned}
$$

elde ederiz. Lemma 2.1, Lemma 2.2 ve Lemma 2.3'deki sonuçları göz önünde bulundurarak yukarıdaki eşitliği

$$
\begin{aligned}
\operatorname{per} W(n) & =F_{n}+F_{n-1}+1+F_{n-1}+1 \\
& =F_{n+1}+F_{n-1}+2
\end{aligned}
$$

şeklinde yazabiliriz. (1) eşitliğinden $F_{n+1}+$ $F_{n-1}=L_{n}$ olduğundan

$$
\operatorname{per} W(n)=L_{n}+2
$$

olur ki bu da istenendir.

Örneğin $n=4$ için

$$
\begin{aligned}
\operatorname{per} W(4)= & \operatorname{per}\left[\begin{array}{llll}
1 & 1 & 0 & 1 \\
1 & 1 & 1 & 0 \\
0 & 1 & 1 & 1 \\
1 & 0 & 1 & 1
\end{array}\right] \\
= & \operatorname{per}\left[\begin{array}{lll}
1 & 1 & 0 \\
1 & 1 & 1 \\
0 & 1 & 1
\end{array}\right] \\
& +\operatorname{per}\left[\begin{array}{lll}
1 & 0 & 1 \\
1 & 1 & 1 \\
0 & 1 & 1
\end{array}\right] \\
& +\operatorname{per}\left[\begin{array}{lll}
1 & 0 & 1 \\
1 & 1 & 0 \\
1 & 1 & 1
\end{array}\right] \\
& =3+3+3=7+2=L_{4}+2
\end{aligned}
$$

olduğunu görebiliriz. 
Şimdi yukarıda elde ettiğimiz sonuçların maple prosedürlerini verelim.

Prosedür A. Aşağıdaki maple prosedür Lemma 2.2'de verilen $G(U(n))$ iki parçalı grafın mükemmel eşlemelerin sayısını hesaplar.

restart:

with(LinearAlgebra):

permanent: $=\operatorname{proc}(\mathrm{n})$

local i,j,r,f,U;

$\mathrm{f}:=(\mathrm{i}, \mathrm{j})->\operatorname{piecewise}(\mathrm{j}-\mathrm{i}=0,1, \mathrm{j}-\mathrm{i}=-1,1, \mathrm{j}-\mathrm{i}=-2,1, \mathrm{j}-$

$\mathrm{i}=\mathrm{n}-1,1,0)$;

$\mathrm{U}:=\operatorname{Matrix}(\mathrm{n}, \mathrm{n}, \mathrm{f})$ :

for $r$ from 0 to $n-2$ do

$\operatorname{print}(\mathrm{r}, \mathrm{U})$ :

for $\mathrm{j}$ from 1 to $\mathrm{n}-\mathrm{r}$ do

$\mathrm{U}[1, \mathrm{j}]:=\mathrm{U}[\mathrm{n}-\mathrm{r}, \mathrm{n}-\mathrm{r}] * \mathrm{U}[1, \mathrm{j}]+\mathrm{U}[1, \mathrm{n}-\mathrm{r}] * \mathrm{U}[\mathrm{n}-\mathrm{r}, \mathrm{j}]$ :

od:

$\mathrm{U}:=$ DeleteRow(DeleteColumn(Matrix(n-r,nr,U),n-r),n-r):

od:

$\operatorname{print}(\mathrm{r}, \mathrm{eval}(\mathrm{U}))$ :

end proc:with(LinearAlgebra):

permanent(n);

Prosedür B. Aşağıdaki maple prosedür Lemma 2.3'de verilen $G(V(n))$ iki parçalı grafın mükemmel eşlemelerin sayısını hesaplar.

restart:

with(LinearAlgebra):

permanent:=proc $(\mathrm{n})$

local i,j,r,g,V;

$\mathrm{g}:=(\mathrm{i}, \mathrm{j})->\operatorname{piecewise}(\mathrm{j}-\mathrm{i}=0,1, \mathrm{i}>1$ and $\mathrm{j}-\mathrm{i}=1,1, \mathrm{j}-$

$\mathrm{i}=-1,1, \mathrm{j}-\mathrm{i}=\mathrm{n}-1,1,0)$;

$\mathrm{V}:=\operatorname{Matrix}(\mathrm{n}, \mathrm{n}, \mathrm{g})$ :

for $r$ from 0 to $n-2$ do

$\operatorname{print}(\mathrm{r}, \mathrm{V})$ :

for $\mathrm{j}$ from 2 to $\mathrm{n}-\mathrm{r}$ do

$\mathrm{V}[1, \mathrm{j}]:=\mathrm{V}[2,1] * \mathrm{~V}[1, \mathrm{j}]+\mathrm{V}[1,1] * \mathrm{~V}[2, \mathrm{j}]$ :

od:
$\mathrm{V}:=$ DeleteRow(DeleteColumn(Matrix(n-r,n$\mathrm{r}, \mathrm{V}), 1), 2)$ :

od:

$\operatorname{print}(\mathrm{r}, \mathrm{eval}(\mathrm{V}))$ :

end proc:with(LinearAlgebra):

permanent(n);

Prosedür C. Aşağıdaki maple prosedür Teorem 2.4'de verilen $G(W(n))$ iki parçalı grafın mükemmel eşlemelerin sayısını hesaplar.

restart:

with(LinearAlgebra):

$\mathrm{P}:=\operatorname{proc}(\mathrm{n})$

local f,H;

$f:=(i, j)->\operatorname{piecewise}(\operatorname{abs}(j-i)<=1,1, \operatorname{abs}(j-i)=n-$

$1,1,0)$;

$\mathrm{H}:=\operatorname{Matrix}(\mathrm{n}, \mathrm{n}, \mathrm{f})$ :

$\operatorname{print}(\mathrm{H})$;

$\operatorname{print}($ Permanent $(\mathrm{H}))$;

end proc:

$\mathrm{P}(\mathrm{n})$;

\section{Sonuç}

Literatürde meşhur sayı dizileri ile iki parçalı grafların mükemmel eşlemelerinin sayısı arasındaki ilişkiyi ortaya koyan birçok çalışma bulabiliriz (Lee ve Lee, 1995; Lee vd., 1997; Lee, 2000; Shiu ve Lam, 2003; Kılıç ve Taşçı, 2007; Kılıç ve Taşçı, 2008; K1lıç ve Stakhov, 2009; Yılmaz ve Bozkurt, 2012; Fonseca vd., 2015; Öteleş, 2017). Bu çalışmalara ek olarak biz de bu makalede, Lucas sayı dizisi ile iki parçalı komşuluk matrisi $(0,1)$-circulant matrisi olan iki parçalı grafın mükemmel eşlemelerinin sayısı arasındaki ilişkiyi gösterdik.

\section{Kaynaklar}

Asratian, A. S., Denley, T. M. J. and Häggkvist, R. 1998. "Bipartite Graphs and their Applications", Cambridge Tracts in 
Mathematics, 131, Cambridge University Press.

Brualdi, R. A. and Gibson, P. M. 1977. "Convex polyhedra of doubly stochastic matrices I: applications of the permanent function", J. Combin. Theory, A 22, 194-230.

Brualdi, R. A. and Cvetkovic, D. 2009. "A Combinatorial Approach to Matrix Theory and Its Applications", CRC Press.

Fonseca, C. M. da, Sogabe, T. and Yilmaz, F. 2015. "Lower k-Hessenberg Matrices and kFibonacci, Fibonacci-p and Pell (p,i) Number", General Mathematics Notes, 31(1), 10-17.

Harary, F. 1969. "Determinants, permanents and bipartite graphs", Mathematics Magazine, 42, 146-148.

Kılıç, E. and Tasçı, D. 2007. "On the permanents of some tridiagonal matrices with applications to the Fibonacci and Lucas numbers", Rocky Mountain Journal of Mathematics, 37(6), 1953-1969.

Kılıç, E. and Tasçı, D. 2008. "On families of bipartite graphs associated with sums of Fibonacci and Lucas numbers", Ars Combinatoria, 89, 31-40.

Kilıç, E. and Stakhov, A. P. 2009. "On the Fibonacci and Lucas p-numbers, their sums, families of bipartite graphs and permanents of certain matrices", Chaos, Solitons\&Fractals, 40(22), 10-21.

Koshy, T. 2001. "Fibonacci and Lucas Numbers with Applications", WileyInterscience, New York.

Koshy, T. 2011. "Fibonacci, Lucas, and Pell numbers, and Pascal's triangle", Mathematical Spectrum, 43(3), 125-132.

König, D. 1915. "Vonalrendszerek és determinások", Math. Termész. Ért., 33, 221229.

König, D. 1916. "Über Graphen und ihre Anwendungen", Math. Annelen, 77, 453465.
Lee, G. Y. and Lee, S. G. 1995. "A note on generalized Fibonacci numbers", The Fibonacci Quarterly, 33, 273-278.

Lee, G. Y., Lee, S. G. and Shin, H. G. 1997. "On the k-generalized Fibonacci matrix Q_\{k\}", Linear Algebra and its Applications, 251, 73-88.

Lee, G. Y. 2000. "k-Lucas numbers and associated bipartite graphs", Linear Algebra and its Applications, 320, 51-61.

Marcus, M. and Minc, H. 1965. "Permanents", American Mathematical Monthly, 72, 577-591.

Minc, H. 1978. "Permanents, Encyclopedia of mathematics and its applications", Addison-Wesley, New York.

Öteleş, A., 2017. "On the number of perfect matchings for some certain types of bipartite graphs", Filomat, 31(5), 4809-4818.

Özkan, E. and Altun, İ, M. 2019. "Generalized Lucas polynomials and relationships between the Fibonacci polynomials and Lucas polynomials", Communications in Algebra, 47(10), 40204030.

Özkan, E., Taştan, M. and Aydoğdu, A. 2018. "2-Fibonacci polynomials in the family of Fibonacci numbers", Notes on Number Theory and Discrete Mathematics, 24(3), 4755.

Özkan, E. and Taştan, M. 2019. "k-Fibonacci Polynomials in The Family of Fibonacci Numbers", Research Reviews: Discrete Mathematical Structures, 6(3), 19-22.

Shiu, W. C. and Lam, P. C. B. 2003. "More on the generalized Fibonacci numbers and associated bipartite graphs", Int. Math., J., 3, 5-9.

The OEIS Foundation Inc., The On-Line Encyclopedia of Integer Sequences, http://oeis.org (Son Erişim Tarihi: 05.03.2020)

Wheland, G. W. 1953. "The Theory of Resonant and its Application to Organic Chemistry", Wiley, New York. 
Yilmaz, F. and Bozkurt, D. 2012. "Some properties of Padovan sequence by matrix methods", Ars Combinatoria, 104, 149-160. 